\title{
Assistente inteligente para auxiliar na prevenção do diabetes tipo 2
}

\author{
Tiago L. Marinho ${ }^{1}$, Wagner W. B. F. Filho ${ }^{1}$, Gabriel F. B. Freire ${ }^{1}$, \\ Mirna de A. Costa ${ }^{2}$, Leandro D. da Silva ${ }^{1}$, Álvaro A. de C. C. Sobrinho ${ }^{3}$, \\ Evandro de B. Costa ${ }^{1}$ \\ ${ }^{1}$ Instituto de Computação - Universidade Federal de Alagoas (UFAL) \\ Maceió - AL - Brazil \\ ${ }^{2}$ Faculdade de Medicina - Universidade Federal de Alagoas (UFAL) \\ Maceió - AL - Brazil \\ ${ }^{3}$ Departamento de Engenharias e Tecnologia \\ Universidade Federal Rural do Semi-Árido (UFERSA) \\ Pau dos Ferros - RN - Brazil \\ $\{$ tlm, wwbff,gfbf, leandrodias, evandro\}eic.ufal.br \\ alvaro.sobrinhodufersa.edu.br
}

\begin{abstract}
Resumo. Neste artigo é apresentado um assistente inteligente para auxiliar na prevenção do diabetes tipo 2. O assistente é baseado em técnicas de inteligência artificial, tomando como base o modelo de um sistema especialista, $e$ representação do conhecimento e do raciocínio de um especialista. O uso de um aplicativo para dispositivos móveis pode favorecer, principalmente, populações de baixa renda e geograficamente remotas, que têm dificuldades de ter acesso a um especialista para acompanhamento contínuo.
\end{abstract}

\section{Introdução}

O Diabetes Melitus (DM), também conhecido como Diabetes, é uma doença crônica e endócrina caracterizada por hiperglicemia, resultante da deficiência do metabolismo da glicose no corpo do indivíduo portador da doença. As consequências a longo prazo da hiperglicemia crônica decorrem de alterações micro e macro vasculares, que levam à disfunção, dano ou falência de vários órgãos. Em 2017, esta doença afetava aproximadamente 425 milhões de adultos, sendo 26 milhões apenas na América do Sul e na América Central, estima-se que até 2045, 629 milhões de pessoas tenham a doença. Além disso, estima-se que 179 milhões de pessoas ainda não diagnosticaram a doença e correm risco de desenvolver maiores complicações com maiores custos, sendo 3.2 milhões de casos no Brasil $^{1}{ }^{2}$. Ainda segundo a IDF, existem evidências de alta qualidade de que a prevenção primária apoia a eficácia de intervenções no estilo de vida na prevenção da progressão de pré-diabetes ao diabetes.

Tomando pelo ponto de vista tecnológico, o presente trabalho se situa no domínio da Computação aplicada a Saúde, envolvendo técnicas de inteligência artificial como elicitação de conhecimento, representação de conhecimento e raciocínio [Russell and Norvig 2010]. Trata-se de um domínio que se iniciou desde a

\footnotetext{
${ }^{1}$ IDF: http://www.diabetesatlas.org/

${ }^{2}$ SBD: https://www.diabetes.org.br/
} 
década de 70 com a proposta do sistema especialista MYCIN, desenvolvido na Universidade de Stanford, nos Estados Unidos, de auxiliar médicos no diagnóstico e tratamento de pacientes com doenças infeciosas no sangue causadas por bactérias [Shortliffe 1977]. Desde então, vários sistemas dessa natureza foram desenvolvidos [Wang et al. 2015, Fioravanti et al. 2011, Rodbard and Vigersky 2011, Wei et al. 2018, Santos et al. 2012]. No entanto, ainda há muito o que evoluir nessa área em busca de sistemas que funcionem a contento e, sobretudo, que cheguem até o usuário final.

Particularmente, para Diabetes, há vários sistemas baseados em conhecimento, implementando funcionalidades que vão do monitoramento ao diagnóstico e proposição de terapia. Neste trabalho, propõe-se tirar proveito desse tipo de tecnologia para ajudar na prevenção de doenças através de sistemas de monitoramento, avaliação e recomendação de conduta aos usuários. O diferencial do aplicativo apresentado neste trabalho é o foco em pré-diabetes, onde praticamente não se tem conhecimento bem estruturado consolidado. Particularmente, aborda-se um problema de modelagem de conhecimento, constituindo-se em uma base de regras de produção do tipo se-então, desenvolvendo-se um motor de inferência que implementa a estratégia de controle denominada encadeamento para frente. Desta forma, o conhecimento especializado é estruturado e disponibilizado em um assistente inteligente para automaticamente ajudar pré-diabéticos na prevenção ou controle, monitorando e analisando periodicamente a situação do usuário a partir da coleta e processamento de dados relacionados a variáveis tais como: peso, circunferência abdominal, índice de massa corporal (IMC), glicemia em jejum, alimentação e pŕatica de exercícios. Além disso, com base na análise realizada pelo aplicativo, este aconselha aos usuários procedimentos preventivos e certos cuidados a se tomar com relação a sua saúde [Leal et al. 2014]. As variáveis e regras de inferência utilizadas para a construção da base de conhecimento foram elencadas e definidas através de reuniões com especialistas e estudos da literatura [Souza et al. 2012] e de sociedades como a IDF e a SBD.

O principal objetivo neste trabalho é disponibilizar um aplicativo para dispositivos móveis para auxiliar na prevenção do Diabetes tipo 2. O foco é, portanto, pessoas com pré-diabetes, e não diabéticos [Souza et al. 2012]. A premissa é de que, como evidencia a IDF, ao sugerir, apoiar e acompanhar mudanças no estilo de vida, é possível diminuir o índice de evolução de pré-diabetes ao diabetes. Iniciativas deste tipo podem ser importantes principalmente para populações de baixa renda e geograficamente remotas, que têm dificuldades de ter acesso a um especialista para acompanhamento contínuo.

\section{Materiais e Métodos}

Ao longo de anos de estudos e pesquisas na área, as regras de auxílio ao diagnóstico foram definidas da seguinte forma:

- Caso o indivíduo tenha quarenta e cinco anos ou mais, ou algum risco aumentado de DM2 (diabetes tipo 2), exame de rastreamento: será verificada a glicemia em jejum.

1. Caso sua glicemia esteja normal, será aconselhado avaliação a cada 3 meses ou conforme o risco do usuário.

2. Caso a glicemia em jejum, esteja entre $100 \mathrm{mg} / \mathrm{dl}$ e $120 \mathrm{mg} / \mathrm{dl}$, será recomendado o TTG (Teste de Tolerância à Glicose). 
3. Caso a glicemia esteja maior ou igual à $126 \mathrm{mg} / \mathrm{dl} \mathrm{em}$ duas ocasiões, ou maior igual a $200 \mathrm{mg} / \mathrm{dl}$, com sintomas, então será diagnosticada a DM2 (Diabetes tipo 2).

- Caso o usuário venha a fazer o TTG (Teste de Tolerância à Glicose), o sistema analisará três opções.

1. Caso a glicemia após duas horas do teste, esteja abaixo de $140 \mathrm{mg} / \mathrm{dl}$, GJA (Glicemia de Jejum Alterada).

2. Caso a glicemia esteja entre $140 \mathrm{mg} / \mathrm{dl}$ e $199 \mathrm{mg} / \mathrm{dl}$, TDG (Tolerância Diminuída à Glicose), então, será recomendado a mudança no estilo de vida (MEV).

3. Caso a glicemia após duas horas do teste, esteja acima de $200 \mathrm{mg} / \mathrm{dl}$, GJA (Glicemia de Jejum Alterada).

Essas são algumas das várias cadeias de raciocínio formadas a partir de condições estabelecidas, representadas no fluxograma para rastreamento e manejo do prédiabetes [Souza et al. 2012], que o sistema processa até gerar um resultado. É verificado, também, se o usuário alcançou as metas ou não. Exemplos de metas são $150 \mathrm{~min} / \mathrm{semana}$ de exercícios, diminuir pelo menos 5 por cento de peso por ano em caso de obesidade, dentre outras.

A aplicação foi construída através da IDE Android Studio ${ }^{3}$, que se utiliza da linguagem de programação Java ${ }^{4}$ e de marcação XML (Extensible Markup Language) ${ }^{5}$. Inicialmente, os dados pessoais e registros médicos dos usuários eram armazenados localmente no dispositivo em um banco de dados SQLite. Em uma versão posterior, foi realizada uma migração do uso de memória persistente do banco SQLite para um armazenamento em nuvem, utilizando o banco de dados Firestore do Firebase, o qual é disponibilizado pela Google ${ }^{6}$.

\section{Aplicação}

Com base no conceito de tomadas de decisão, o problema foi modelado sobre uma estrutura de árvore em que cada informação do paciente como, por exemplo idade, nível de glicemia, resultado de exames TTG, etc, é um estado, que será analisado e processado um diagnóstico. A aplicação é modelada com o padrão de arquitetura MVC (Model, View e Controller). Além disso, foi utilizado a IDE (Ambiente de desenvolvimento integrado) Android Studio (linguagem de programação Java), SQLite e Firestore, como bancos de dados da aplicação.

A arquitetura MVC permite que o desenvolvimento do projeto fique mais fácil, pois organiza o projeto em camadas, cada um como uma sua função específica no sistema. Desse modo, em relação à modelagem da aplicação com persistência de dados, leitura, escrita e validações, competem a camada "Model". O módulo "Controller", é responsável por ser uma "interface"entre a interação do usuário e os dados do sistema. Ele comanda todas as informações das variáveis dos usuários, como peso, altura, IMC,

\footnotetext{
${ }^{3}$ https://developer.android.com/studio

${ }^{4}$ https://www.java.com/pt_BR/download/

${ }^{5}$ https://www.w3.org/XML/

${ }^{6}$ https://firebase.google.com/docs/firestore/
} 


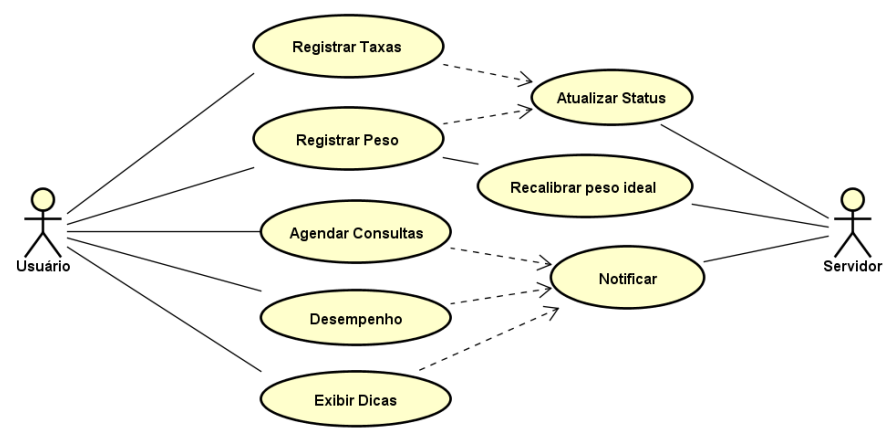

Figura 1. Casos de Uso
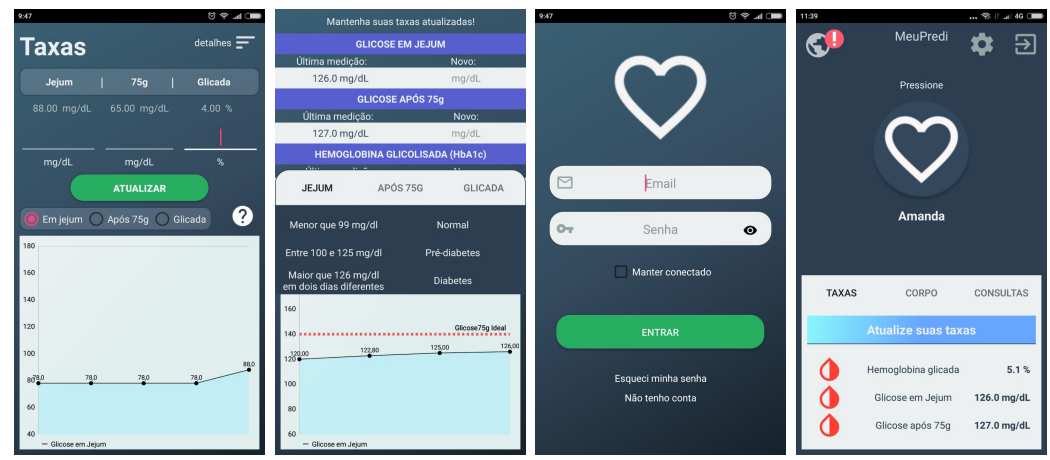

Figura 2. Telas de taxas, informações, login e de perfil

glicose em jejum, etc. Para o armazenamento dessas variáveis no banco de dados, é utilizado o módulo "Controller". O módulo "View"foi responsável pela administração das telas do sistema, de forma que motivasse a interação do usuário com a aplicação. O diagrama de casos de uso ilustrado na Figura 1 é uma descrição do cenário que mostra as funcionalidades do sistema a partir do ponto de vista do usuário.

Finalmente, o motor de inferência foi implementando de forma que seja flexível em caso de mudanças. Em uma aplicação feita na linguagem Java e utilizando o Android Studio, é vantajoso guardar a base de conhecimento, no caso o conjunto de regras, em um arquivo distinto. No caso de um sistema especialista voltado para a prevenção de diabetes, caso haja inclusão de novas variáveis, é possível modificar o motor de inferência com mais facilidade. No caso desta aplicação, a base de conhecimento foi em torno do fluxograma para rastreamento e manejo do pré-diabetes [Souza et al. 2012].

O sistema permite que o usuário cadastre suas taxas, de forma que ele seja sempre informado através de um gráfico como pode ser visto na Figura 2, informando suas taxas e datas cadastradas. Além disso o sistema também permite que o usuário cadastre seus pesos e circunferência abdominais (Figura 3), já que são variáveis que podem influenciar na DM2. De forma a motivar o uso da aplicação, também é permitido que o usuário informe suas consultas, detalhando seu local, hora e data, de forma que a aplicação emita alertas para o usuário ao longo do tempo. 

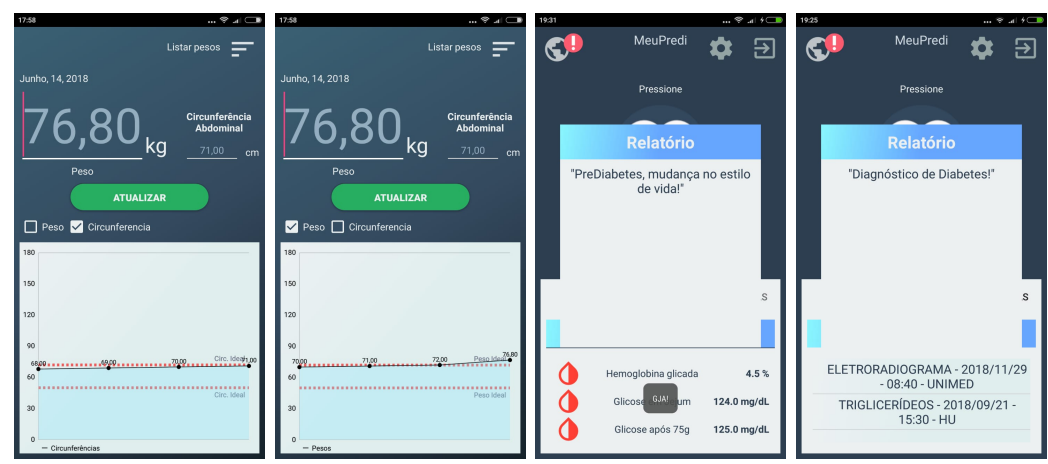

Figura 3. Telas de circunferência, peso e diagnóstico

Na tela de taxas, é possível que o usuário cadastre suas informações relacionadas à glicose e acompanhe seu histórico através de um gráfico. Caso o usuário queira ter uma noção sobre as informações, ao clicar no ícone da estrela, é mostrado a ele informações em relação à cada taxa, como mostrado na Figura 2 ao lado direito. A linha trastejada em vermelho, no gráfico, é o limite da glicose do usuário para que ela continue normal, acima disso, ele pode ser considerado um pré-diabético, como pode ser visto na Figura 2. De forma análoga à tela de taxas, na tela de pesos e circunferências, também é possível fazer o cadastro destas variáveis e acompanhá-las através de um gráfico. Um pouco diferente da tela de taxas, neste caso, a linha trastejada delimita os valores onde o IMC (índice de massa corporal) do usuário é considerado normal, acima disso é considerado acima do peso, abaixo, considerado abaixo do peso. O mesmo é mostrado no gráfico relacionado à circunferência abdominal, a linha trastejada delimita uma circunferência considerada normal para o usuário. Estes dois casos são demonstrados na Figura 3.

Após o cadastro das taxas, de acordo com os cálculos mostrados no raciocínio, na seção 2.1, a aplicação exibe um diagnóstico informando se o usuário está com a glicose normal, se já é considerado um pré-diabético ou um diabético, como mostrado na Figura 3. Outra forma de motivar o usuário no uso da aplicação, é permitir o cadastro de suas próximas consultas e o sistema notifique quando elas estiverem em datas próximas. É possível cadastrar o local, data, horário e uma descrição sobre a consulta. Além disso, foi considerado como uma forma de motivação, dar dicas de saúde como, por exemplo, da água pois é algo que não tem restrição para nenhum usuário. Essas telas não são mostradas devido a limitação de espaço.

\section{Conclusão}

A Diabetes Mellitus é uma doença crônica que resulta na deficiência do metabolismo da glicose no corpo do indivíduo. As suas consequências a longo prazo podem acarretar danos ou falência de vários órgãos. Sendo assim, a existência de um aplicativo auxiliar portátil e de fácil uso se revela como uma forte ferramenta na prevenção e no controle da doença. Uma forma de evitar a evolução do quadro de pré-diabetes para diabetes é incentivar o usuário a ter um estilo de vida mais saudável, baseado em atividades físicas e controle da alimentação. O aplicativo funciona como uma ferramenta de uso contínuo, capaz de acompanhar o usuário por longos períodos, registrando medições e gerando gráficos pertinentes ao controle das taxas e a manutenção da saúde do usuário. Além disso, apresenta dicas e informações úteis a longo prazo, visto que é possível traçar um histórico de 
evolução, o que muitas vezes não é possível no ambiente residencial, principalmente em populações com difícil acesso ao especialista, como de baixa renda ou geograficamente remotas. Como trabalhos futuros, duas principais áreas de atuação estão em desenvolvimento. Primeiro, a implementação de novos módulos como, por exemplo, dicas de saúde, troca de informações utilizando o padrão HL7-CDA ${ }^{7}$, dentre outros. A segunda área é a realização de estudos clínicos no Hospital Universitário da UFAL apoiados por estudantes de medicina e médicos. Está em fase final de edição o projeto para ser submetido ao comitê de ética através da Plataforma Brasil ${ }^{8}$ para realização do estudo clínico do projeto. Também está em desenvolvimento um estudo de usabilidade do aplicativo.

Agradecimentos: O presente trabalho foi realizado com apoio do Conselho Nacional de Desenvolvimento Científico e Tecnológico (CNPq), e da Universidade Federal de Alagoas (UFAL).

\section{Referências}

Fioravanti, A., Fico, G., Arredondo, M. T., and Leuteritz, J. . (2011). A mobile feedback system for integrated e-health platforms to improve self-care and compliance of diabetes mellitus patients. In 2011 Annual International Conference of the IEEE Engineering in Medicine and Biology Society, pages 3550-3553.

Leal, L. B., Moura, I. H., de Carvalho, R. B. N., Leal, N. T. B., Silva, A. Q., and da Silva, A. R. V. (2014). Qualidade de vida relacionada à saúde de pessoas com diabetes mellitus tipo 2. Revista da Rede de Enfermagem do Nordeste, 15:676-682.

Rodbard, D. and Vigersky, R. A. (2011). Design of a decision support system to help clinicians manage glycemia in patients with type 2 diabetes mellitus. Journal of Diabetes Science and Technology, 5(2):402-411.

Russell, S. and Norvig, P. (2010). Artificial Intelligence: A Modern Approach. Series in Artificial Intelligence. Prentice Hall, Upper Saddle River, NJ, third edition.

Santos, K., Feistauer, L., Carvalho, M., Silva, L., and Rezende, K. (2012). Sisped 2.0: An extension of a system to monitor diabetic patients. In 2012 6th Euro American Conference on Telematics and Information Systems (EATIS), pages 1-8.

Shortliffe, E. H. (1977). Mycin: A knowledge-based computer program applied to infectious diseases. In Proceedings of the Annual Symposium on Computer Application in Medical Care, page 66-69.

Souza, C. F. d., Gross, J. L., Gerchman, F., and Leitão, C. B. (2012). Pré-diabetes: diagnóstico, avaliação de complicações crônicas e tratamento. Arquivos Brasileiros de Endocrinologia e Metabologia, 56:275 - 284 .

Wang, L., Pedersen, P. C., Strong, D. M., Tulu, B., Agu, E., and Ignotz, R. (2015). Smartphonebased wound assessment system for patients with diabetes. IEEE Transactions on Biomedical Engineering, 62(2):477-488.

Wei, S., Zhao, X., and Miao, C. (2018). A comprehensive exploration to the machine learning techniques for diabetes identification. In 2018 IEEE 4th World Forum on Internet of Things (WF-IoT), pages 291-295.

\footnotetext{
${ }^{7}$ http://www.hl7.org

${ }^{8} \mathrm{http}: / /$ plataformabrasil.saude.gov.br/login.jsf
} 